Int. J. Dev. Biol. 58: 775-781 (2014)

doi: $10.1387 / \mathrm{ijdb} .140277 \mathrm{jt}$

\title{
Epithelial cell division in the Xenopus laevis embryo during gastrulation
}

\author{
GUILLAUME HATTEE ${ }^{1,2}$, MARC TRAMIER ${ }^{1,2}$, CLAUDE PRIGENT ${ }^{1,2}$ and JEAN-PIERRE TASSAN*,1,2 \\ ${ }^{1}$ CNRS UMR 6290 and ${ }^{2}$ Université de Rennes 1, Institut de Génétique et Développement de Rennes, \\ Rennes, France
}

\begin{abstract}
How vertebrate epithelial cells divide in vivo and how the cellular environment influences cell division is currently poorly understood. A sine qua non condition to study cell division in situ is the ease of observation of cell division. This is fulfilled in the Xenopus embryo at the gastrula stage where polarized epithelial cells divide with a high frequency at the surface of the organism. Recently, using this model system, we have shown that epithelial cells divide by asymmetric furrowing and that the mode of cell division is regulated during development. Here, we further characterize epithelial cell division in situ. To this end, we used confocal microscopy to study epithelial cell division in the ectoderm of the Xenopus laevis gastrula. Cell division was followed either by indirect immunofluorescence in fixed embryos or by live imaging of embryos transiently expressing diverse fluorescent proteins. Here, we show that during cytokinesis, the plasma membranes of the two daughter cells are usually separated by a gap. For most divisions, daughter cells make contacts basally at a distance from the furrow tip which creates an inverted teardrop-like shaped volume tightly associated with the furrow. At the end of cytokinesis, the inverted teardrop is resorbed; thus it is a transient structure. Several proteins involved in cytokinesis are localized at the tip of the inverted teardrop suggesting that the formation of the gap could be an active process. We also show that intercalation of neighboring cells between daughter cells occasionally occurs during cytokinesis. Our results reveal an additional level of complexity in the relationship between dividing cells and also with their neighboring cells during cytokinesis in the Xenopus embryo epithelium.
\end{abstract}

KEY WORDS: cytokinesis, asymmetric furrowing, contractile ring, anillin, actin, myosin

\section{Introduction}

Cytokinesis is the process by which dividing cells separate to produce two daughter cells. It is driven by a contractile ring composed of actin and myosin. It is commonly accepted that this ring is established perpendicularly to the mitotic spindle and is placed at equidistance of the mitotic chromosomes allowing their equal distribution to two daughter cells (Almonacid and Paoletti, 2010, Rappaport, 1996). The precise spatio-temporal regulation of the ring ensures the proper cell separation which otherwise could ultimately lead to abortive cytokinesis (Normand and King, 2010). Although a detailed mechanism of action is currently unknown, it is established that the actomyosin-based contractile ring produces the force necessary for ingression of the plasma membrane between the two daughter cells (Fededa and Gerlich, 2012). In addition to actin and myosin, multiple proteins are necessary to cytokinesis to proceed (Green et al., 2012). Anillin, a large multidomain protein is involved in the spatial positioning of the cleavage furrow during cytokinesis (Piekny and Maddox, 2010). Various anillin domains allow its interaction with cytokinetic proteins including actin, myosin and septin. In absence of anillin, the cytokinetic ring still forms, however it is not stably positioned. In isolated in vitro unpolarized cultured cells, the contraction of the contractile ring appears approximately concentric.

Vertebrate cell division is mostly studied in in vitro cultured cells. For example, isolated cells are particularly well suited for high-throughput screen (Kittler et al., 2004). Although, studies using the in vitro cultured cells have led to the discovery of basic mechanisms governing cytokinesis, it appears paradoxical that the majority of information on cell division of multicellular organisms

Abbreviations used in this paper: MHC, myosin II heavy chain.

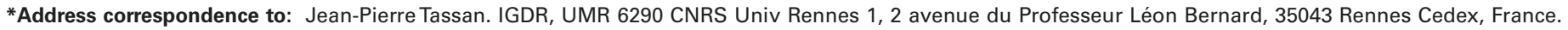
E-mail: jean-pierre.tassan@univ-rennes1.fr
}

Supplementary Material (3 movies and 3 figures) for this paper is available at: http://dx.doi.org/10.1387/ijdb.140277jt

Accepted: 7 December 2014

ISSN: Online 1696-3547, Print 0214-6282 
comes from observations of isolated cells. While observations and analyses of some aspects of cell division can be facilitated in vitro, some events can also be artificially amplified. For example, when they enter mitosis, in vitro cultured cells undergo remarkable shape changes. Contacts of the mitotic cells with the substratum are usually attenuated and cells round up dramatically (Cramer and Mitchison, 1997, Maddox and Burridge, 2003). In intact tissues, such as epithelium, in which cell-cell contacts are tight, modifications of the mitotic cell shape still occur. However, they are less pronounced probably because neighboring cells oppose to mitotic cell deformation.

Epithelia act as physical and selective chemical barriers between distinct body compartments; they can also constitute a border separating the organism from the external environment. It appears essential to maintain this function also during cell division. In an epithelium, cells are subjected to various constraints including cell polarity and external physical forces generated by neighboring cells deformations and morphogenetic movements, especially in the case of a developing embryo. These constraints could likely impact on the mode of cytokinesis. The limited numbers of vertebrate model systems that can be used to study epithelial cell division in vivo is a major difficulty leading to poor understanding of these mechanisms acting in situ. Following division of vertebrate epithelial cells in situ is particularly difficult because epithelia are usually embedded deep in the organism. The early Xenopus embryo is an excellent model to study cytokinesis of epithelial cells in vivo (Kubiak et al., 2014). Indeed, the superficial cell layer of the Xenopus gastrula is constituted of epithelial cells allowing easy observation in situ. The epithelium is established very soon after fertilization. During Xenopus development, establishment of cell junctions including the tight junctions (TJs) occurs a few cell cycles after fertilization (Merzdorf et al., 1998). Also, it was shown that the position of the TJs in the baso-apical axis changes during the embryo development
(Merzdorf et al., 1998). An additional advantage of the Xenopus embryo is its high mitotic index during the early development.

Using the Xenopus early embryo as a model system, we have previously shown that the cytokinetic furrow ingresses asymmetrically and that the mode of epithelial cells division changes during development of Xenopus embryos (Le Page et al., 2011). Indeed, during the early period of development, the furrow ingresses from the apico-basal direction, while later the plasma membrane ingresses in the baso-apical direction. The asymmetric ingression of the cytokinetic furrow is therefore in sharp contrast with the approximate concentric ingression observed in isolated cells. However, the asymmetric furrowing appears to be the mode of epithelial cells division in invertebrates and vertebrates model systems including nematodes (Audhya et al., 2005), Drosophila (Founounou et al., 2013; Guillot et al., 2013; Herszterg et al., 2013; Morais-de Sa et al., 2013) and mouse intestine (Jinguji and Ishikawa, 1992).

In a previous study focused on MELK (Maternal Embryonic Leucine zipper Kinase), i.e. a mitotic protein kinase involved in cytokinesis, we observed in fixed embryos that at the leading edge of the cytokinetic furrow the plasma membranes of the two daughter cells are separated by a gap (Chartrain et al., 2013). Because the presence of this gap was puzzling and to our knowledge was not characterized, we further analyzed it using in vivo imaging in the Xenopus early embryo and present here these results.

\section{Results}

\section{Agap transiently forms between the two daughter cells during cytokinesis of epithelial cells in the Xenopus embryo}

In a previous study, we observed the formation of a gap between plasma membranes of the two daughter cells of dividing epithelial cells in the Xenopus gastrula ectoderm (Chartrain et al., 2013). Indirect immunofluorescence using specific anti-xMELK antibod-
A

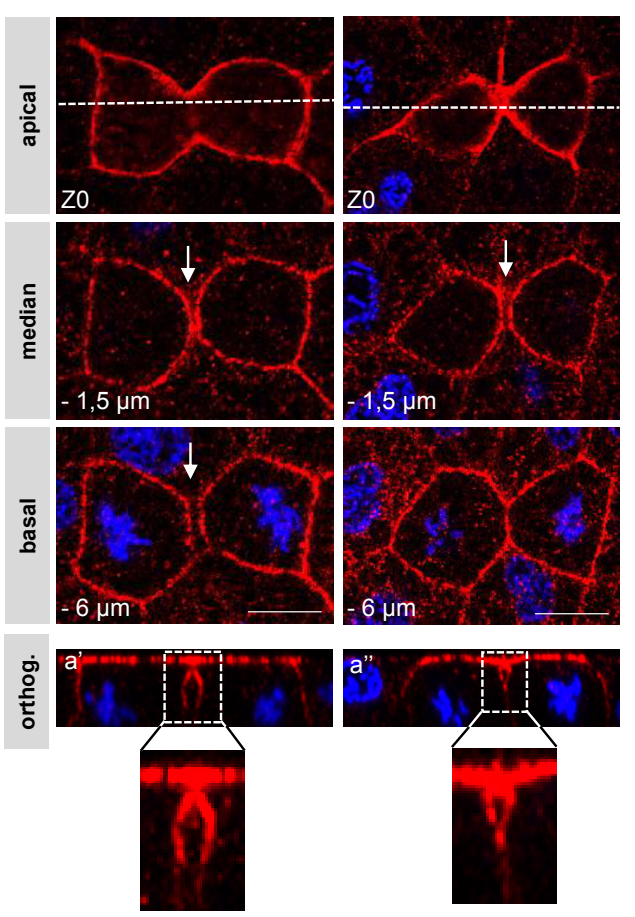

B

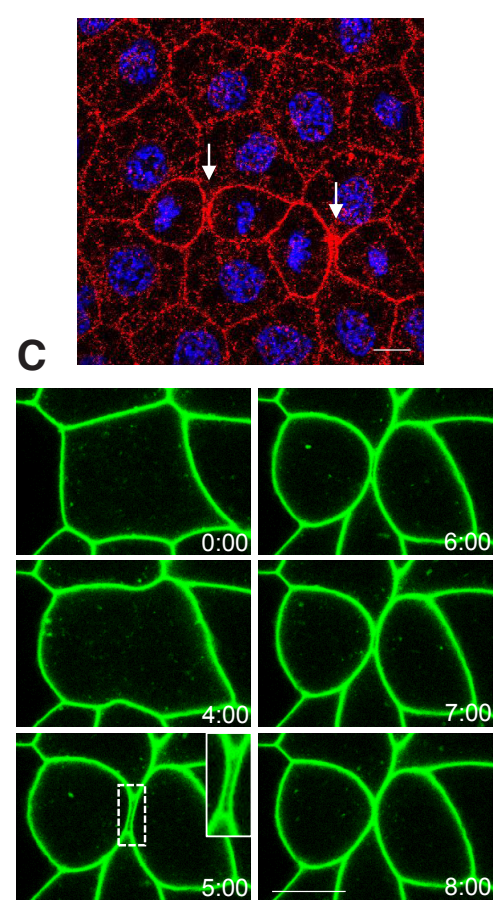

Fig. 1. During epithelial cell cytokinesis in the Xenopus gastrula, a gap transiently forms between the plasma membranes of the two daughters cells. (A,B) Albino embryos were fixed at the gastrula stage and indirect immunofluorescence was performed with anti-xMELK antibodies (red and stained for DNA with TO-PRO-3 (blue). (A) Three optical sections are shown An orthogonal projection along the daughter cells axis perpendicular to the cytokinetic furrow (white dashed line) is shown (orthog.). High magnifications of the insets in orthogonal projections ( $a$ ' and $a^{\prime \prime}$ ) are shown. On the left column, the gap between daughter cells is visible over the entire height of daughter cells wheras on the right daughter cells are in contacts basally to the gap thus closing the gap and creating an inverted teardrop-like shape volume. (B) A wide-field of the epithelium presented in A showing two cytokinetic cells surrounded by interphase cells. White arrows point on the gap between the two daughter cells. Such a gap is not detected between interphase cells. (C) Time-lapse imaging of a dividing cell expressing GFP$G P I$. Embryos were microinjected in one blastomere at the two cells stage with mRNA encoding GFP-GPI and observed when they reached the gastrula stage 11. A high magnification of the gap is shown in the insert. Time is given in minutes. 0 is defined as the time before any sign of membrane contraction can be detected. Scale bars: 10 um. 
Fig. 2. Three types of epithelial cell cytokinesis in Xenopus laevis gastrula. Live-imaging of GFP-GPI, green, $(\mathbf{A}, \mathbf{B})$ and GFP GPI+RFP-GPI, merge of green and red, (C) expressing embryos showing a dividing cell with the inverted teardrop between the daughter cells and which progresses with the cleavage furrow (A), a cell which divides without formation of a gap (B), and neighboring cells which intercalate between the two daughter cells during cytokinesis (C). Two $(\mathbf{A}, \mathbf{C})$ or three (B) confocal planes are shown. Orthogonal projections along the daughter cell axis (white dashed lines) are shown in $(\mathbf{A}, \mathbf{B})$. In the orthogonal projection in (A), a high magnification of the teardrop is shown in the insert. Arrowhead over the orthogonal projection in (B) indicates that the furrow ingresses asymmetrically from the apical membrane towards the basal region. In (C) two orthogonal projections respectively along the daughter cells axis (d) and the neighboring cells axis (n, grey dashed line) are shown. The white arrow on the orthogonal projection (d) points on the two neighboring cells which intercalate between the two daughter cells. Time is given in minutes. 0 is defined as the time before any sign of membrane contraction can be detected. Scale bars: $10 \mu m$.

ies in trichloroacetic acid (TCA) fixed embryos shows that XMELK is localized at the cell periphery in interphase cells and further accumulates in cytokinetic cells (Fig. 1A, 1B and Le Page et al., 2011). This also shows that the baso-lateral membrane of the cytokinetic cell is already divided whereas the apical membrane is not yet divided (Fig. 1A, compare confocal plane at Z0 and $-6 \mu \mathrm{m}$ ). Indeed, we have previously shown that the cytokinetic furrowing in epithelial cells of gastrula progresses asymmetrically from the basal membrane towards the apical membrane. Remarkably, a gap between the two daughter cells is evident (Fig. 1A, arrows). This gap appears only in cytokinetic cells and was never detected between interphase cells (larger field in Fig. 1B). This suggested that the gap could be transient. In the vast majority of cases, the gap was observed over the entire height of daughter cells (Fig. 1A, orthogonal projections a'). However, in rare cases we observed that daughter cells were in close contact basally to the gap (Fig. $1 \mathrm{~A}$, orthogonal projections a"). In this configuration, the gap has a characteristic inverted teardrop like shape. The reason why the gap appears variable in shape is yet unknown but we hypothesized that it could be linked to the method used to fixe embryos. Indeed, in embryos fixed with formaldehyde instead of TCA the gap is not detectable and the two daughter cells appear in close contact over their entire height (data not shown). Therefore, we tested if the gap was observable in living embryos. To visualize plasma membranes, we used a GFP fused with a glycosylphosphatidylinositol (GPI)-anchor addition sequence (GFP-GPI). The GPI modification allows anchoring of the modified proteins to the plasma membrane of all interphase and dividing cells. Division of epithelial cells was followed by time-laps confocal microscopy (Fig. 1C, the same confocal plane is shown over time). Cytokinesis causes extensive remodeling of the cytokinetic cell which rapidly rounds up (Fig. 1C, times 0 to 4 minutes). At 4 minutes the plasma membrane begins to bend indicating that cytokinesis has started. One minute later, the two daughter cells are divided; a gap between them appears and continues to be visible during the next 2 minutes. The gap frontiers are delineated by plasma membranes of the two daughter cells and the neighboring cells (high magnification in Fig. 1C). Over time, the gap usually becomes smaller until the two daughter cells are in close contact. During resorption of the gap, plasma membranes are highly fluctuating showing movement towards and away from each other (Movies 1 and 2). These breathing oscillations indicate that the gap closure is not a continuous process and suggests that it could proceed by contraction and relaxation waves. As with fixed embryos, observations over a wide field show that this gap is specific of dividing cells (Supplementary Fig. S1). Taken together, our results show that during cytokinesis the plasma membranes of the two daughter cells are transiently spaced from one another.

The gap between daughter cells follows the cleavage furrow

In living embryos, the gap between the two daughter cells has an inverted teardrop-like shape (Fig. 2A, orthogonal projection). While the apical membrane is not yet divided, the daughter cells usually make new contacts with one another at their baso-lateral membrane. Therefore, the inverted teardrop is apically delineated 
by the ingressing membrane and basally by new contacts formed by plasma membranes of the two daughter cells (Fig. 2A, high magnification). Our analysis shows that $97 \%$ of cytokinetic cells $(n=55)$ divide with this configuration. Moreover, orthogonal projections show that the inverted teardrop progresses with the tip of the cleavage furrow (Fig. 2A). This indicates that the inverted teardrop is intimately linked to the cytokinetic process. At the end of cell division, the teardrop is resorbed, thus explaining why it is transient. Although infrequent, in some cells of TCA-fixed embryos, the teardrop can also be observed. Therefore, TCA fixation may not efficiently preserve the new contacts which could explain why the gap remains open in fixed embryos while it is closed for the majority of living cells. Taken together, our results show that in the vast majority of cases, the two daughter cells make new contacts with one another below the gap thus creating an inverted teardrop.

In addition to this major configuration, we determined that $3 \%$ of dividing cells do not exhibit a gap between daughter cells (Fig. $2 \mathrm{~B})$. However, this cytokinesis is particular because the cleavage furrow progresses asymmetrically in the apico-basal direction (arrowhead on the orthogonal projection) whereas in most cases at this developmental stage, cytokinesis proceeds from the basal towards the apical membrane (Le Page et al., 2011). Correlatively, the lateral contraction was also limited particularly on the apical side in these particular cells (Fig. 2B). The fate of these cells is unknown yet and the reason why they divide following this mode of cytokinesis will also need to be clarified.

Microinjection of an mRNA into embryos leads to its mosaic

A

$$
\begin{gathered}
\text { A GFP-Anillin, } \\
\text { RFP-GPI }
\end{gathered}
$$
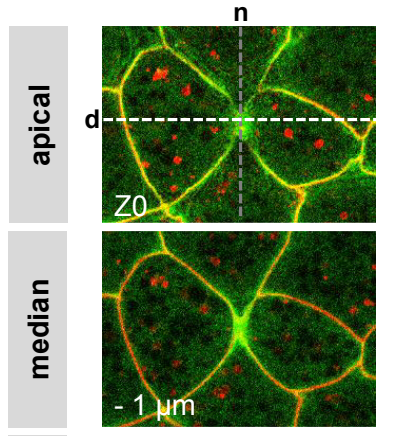

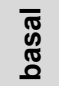
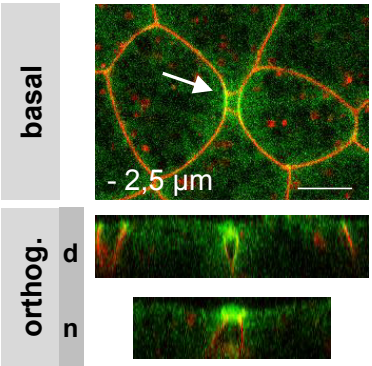

B

Actin: mcherry-Utr-CH
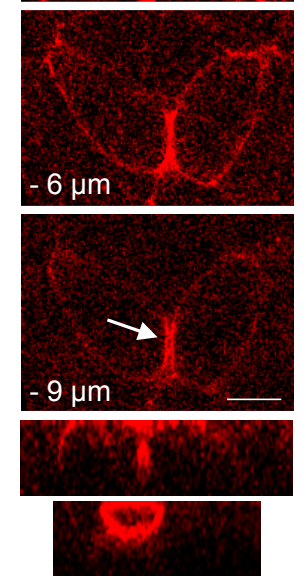

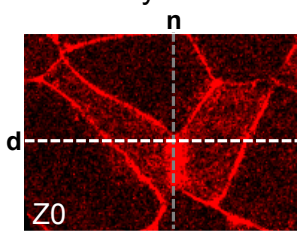

C MHC: SF9-GFP
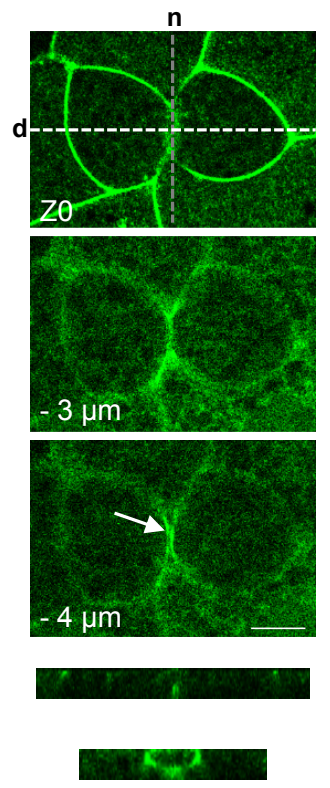

Fig. 3. Anillin, actin and myosin II heavy chain (MHC) localize at the leading edge of the inverted teardrop during epithelial cell cytokinesis in gastrula. Localizations of GFP-anillin + RFP-GPI (respectively green and red, a merge is presented) (A), actin detected with the mcherry-Utr-CH probe (B), and myosin II heavy chain (MHC) using the SF9-GFP intrabody (C). For each, 3 confocal planes and orthogonal projections along the daughter cells (d) and neighboring cells ( $n$ ) axes are shown. The arrows point on the gap between the two daughter cells. Scale bars, $10 \mu \mathrm{m}$. expression in terms of levels and spatial distribution. The slow diffusion of the mRNA from the injection point compare to the very rapid cell division cycle limits its spreading to a confined region of the embryo. Thus, the progeny of the injected cell produces a clone-like cell group. This is consistent with our results showing that the two daughter cells usually make new contacts with one another while the apical membrane is not yet divided. This mechanism allows keeping cells in close contact with one another and can explain how the clone-like cells group remains compact. Interestingly, at the clone periphery some cells or small cell groups appear as isolated from the clone by cells belonging to another progeny (an example is shown in Fig. S2). This could be explained by migration of cells which would escape from the clone and infiltrate into neighboring clones. However, we never observed such a long-range cell migration in the epithelium plane. It has been shown that remodeling of cell-cell contacts allows cell intercalation during convergent extension during the Xenopus embryo elongation (Keller, 2002). As an alternative, we hypothesized that concomitantly with cytokinesis, neighboring cells could intercalate between the two daughter cells. If this mechanism exists then it could explain how some cells at the periphery of the clone become separated by cells which do not belong to the clone. This mechanism could participate in creating the mosaicism. To test this hypothesis, we injected 2 cells of 4 cells stage embryos with GFP-GPI and the two other cells with RFP-GPI. The two colors expression allows following the green and red cells and specifically detecting intercalation events at the border between the two cell groups. Figure $2 \mathrm{C}$ shows two green cells separated by a red cell (t 0$)$. As the red cell divides and constricts apically, the plasma membranes of the two green neighboring cells interdigitate. They finally join at the division site and make contact with one another (t 7:30). Then the contacts propagate baso-laterally (t 12:00). Those daughter cells contacts were observed during several hours (Movie 3) indicating that the intercalation was stable over time. Another example is shown in Fig. S3. We also observed that neighboring cells transiently interdigitated between the two daughter cells with contacts lasting several minutes (not shown). Taken together, our results show that cytokinesis provide the conditions for cell intercalation and thus participate to cell mixing in the ectodermal epithelium of Xenopus gastrula.

\section{Anillin, actin and myosin are present at the tip of the inverted teardrop}

As the gap and the inverted teardrop follow the cleavage furrow, we investigated a potential relationship of the two structures by following the localization of several proteins involved in cytokinesis. This includes anillin using a GFP-anillin fusion construct, endogenous actin using the Utr-Ch-mcherry probe (Burkel et al., 2007) and the endogenous myosin II heavy chain (MHC) using the SF9-GFP intrabody (Nizak et al., 2003). As shown by orthogonal projections along the neighboring cells axis (orthog. $n$ in Fig. $3 A, B$ and $C$ ), the 3 proteins are localized on the contractile cytokinetic ring. Interestingly, orthogonal projections along the daughter cells axis show that GFP-anillin is also localized at the leading edge of the inverted teardrop labelled by RFP-GPI (orthog. $d$ in Fig. 3A). Similarly, actin and MHC also decorate the 
A

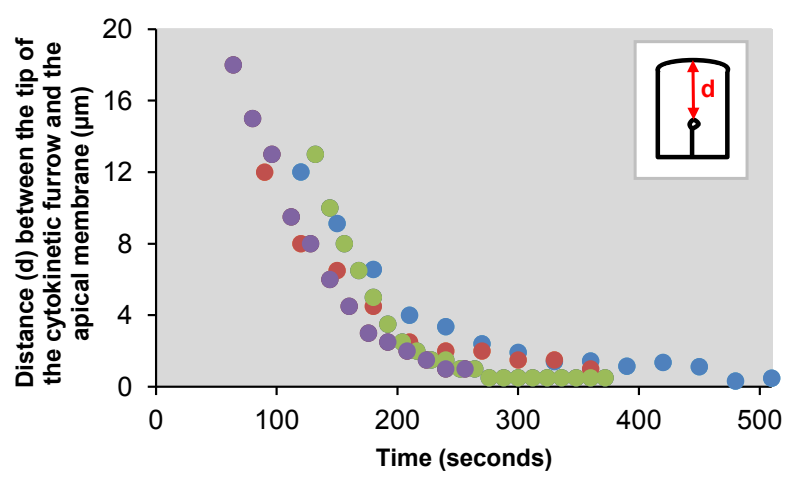

B

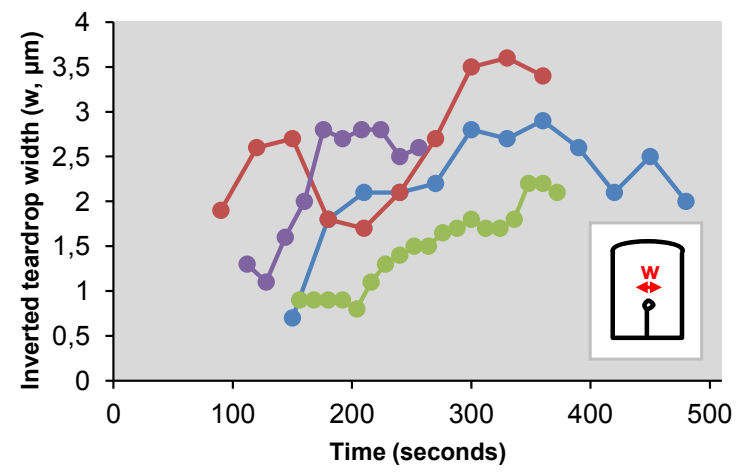

Fig. 4. Dynamics of the cleavage furrow during epithelial cell cytokinesis. (A) Graphs plotting the distance between the cytokinetic furrow and the apical membrane over time $(n=4)$ showing that cytokinesis is initially rapid and becomes slower when the furrow is 2.5-4.5 $\mu \mathrm{m}$ of the apical membrane. (B) Quantification of the gap width during the fast and the slow phases progression of the cytokinetic furrow over time. The same cells as in (A) were analyzed.

inverted teardrop (orthog. $d$ in Fig. 3 B and C). These results suggest that the plasma membrane separation below the cytokinetic furrow could be an active process and involves cytokinetic proteins.

\section{Dynamics of the cleavage furrow ingression}

We have previously shown that epithelial cells divide by asymmetric furrowing (Le Page et al., 2011). Because these cells are polarized, we asked the question whether ingression of the cleavage furrow could differ upon its position along the baso-apical axis. Quantitative analysis of cleavage furrow progression over time shows that it progresses with 3 kinetics (Fig. 4A, kinetics for 4 representative cells are shown). Until it reaches 2.5 to $4.5 \mu \mathrm{m}$ from the apical membrane, the furrow progresses rapidly $(6.8+/-2.1 \mu \mathrm{m} /$ min.). After a transition, its speed becomes slower $(1.1+/-0.6 \mu \mathrm{m} /$ min.) until the end of cytokinesis. We also quantified the inverted teardrop width over time (Fig. 4B). Consistent with the breathing oscillations described above (Movie 1), the teardrop width appears more heterogeneous over time. During the rapid progression of the cleavage furrow, the teardrop maximum width is about $1.76 \mu \mathrm{m}$ $+/-0.78(n=10)$. Its lifetime, as defined by the time it is observed at a constant position in the baso-apical axis, is of the order of one minute (data not shown and Fig. 2A). In contrast, after slowing, the gap is significantly greater, about $2.71 \mu \mathrm{m}+/-0.75(n=10)$ and its lifetime is much prolonged. Indeed, it takes about 4.5 minutes to disappear. Taken together, our results show that during epithelial cell cytokinesis, a gap usually forms between the two daughter cells. This gap progresses together with the cleavage furrow which is itself submitted to two progression speed along the apico-basal axis. In their majority, the two daughter cells make contacts at the baso-lateral side thus creating an inverted teardrop-like volume, the size of which also changes along the apico-basal axis.

\section{Discussion}

Using the Xenopus embryo as a model system to study cytokinesis in a vertebrate organism, we have previously shown that epithelial cells divide by asymmetric furrowing (Le Page et al., 2011). In the present study, we further characterized asymmetric furrowing. We show that at the leading edge of the division furrow, the plasma membranes of the two daughter epithelial cells are separated by a gap. In the vast majority of cases, the two daughter cells make new contacts a few microns basally to the furrow tip thus creating an inverted teardrop-like shape volume. This general outline is however the subject of some variations such as intercalation of the neighboring cells between the two daughter cells.

How the gap forms is yet unknown, however our results show that proteins involved in cytokinesis including anillin, actin and myosin II heavy chain are localized at the tip and laterally of the inverted teardrop. This result suggests that membrane spreading could be an active process. This consequently raises the question why the daughter cells membranes are transiently separated by a gap during cytokinesis. Several, not exclusive, hypothesis can be proposed. The leading edge of the cytokinetic furrow could correspond to the anchoring of the cytokinetic ring to the plasma membrane allowing its ingression. This could require or induce the plasma membrane specific bending at the leading edge of the cytokinetic furrow. The teardrop is reminiscent to the furrow canal observed during cellularization of the Drosophila embryo (Lecuit and Wieschaus, 2000). As in the Xenopus embryo, it has been shown that the furrow canal accumulates cytokinetic molecules but contrary to the teardrop, the furrow canal is not anchored to a transversal cytokinetic ring. This suggests that if the teardrop and the furrow canal are similar structures, the Xenopus teardrop shape may not be solely dictated by the cytokinetic ring. In addition to ingression, cytokinesis requires addition of new membranes. In the Xenopus one cell embryo it has been shown that the leading edge of the cytokinetic furrow is mainly inherited from the surface plasma membrane (Byers and Armstrong, 1986). In this study, a teardrop-like structure is visible at the tip of the cytokinetic furrow in dividing two cells embryos. In contrast, the lateral side of the furrow is mainly constituted by new membranes. One can hypothesize that membrane spreading at the leading edge of the cytokinetic furrow allows creating two independent membrane domains where new membranes are added. This raises the attractive possibility that cytokinetic proteins are localized at the leading edge of the inverted teardrop to produce the forces necessary to limit membrane spreading.

Our results show that the cytokinetic furrow speed decreases when it is 2.5 to $4.5 \mu \mathrm{m}$ from the apical membrane. This distance is consistent with the position of the apical junctional complex (Woolner and Papalopulu, 2012). The size of the inverted teardrop concomitantly increases during its progression towards the apical membrane. This could be correlated with the increasing density of cellular junctions and the associated cytoskeleton at the apical 
side. Therefore, puling forces which spread plasma membranes could be higher when the cytokinetic furrow riches the apical adhesion belt. During cytokinesis, two perpendicular actin-based cytoskeletons must cross: one associated with the apical adhesion complexes in the epithelium plan and the contractile cytokinetic ring orthogonal to the epithelium plan. How these two cytoskeletons are untangled is yet unknown but remodeling of the apical actin cytoskeleton may be necessary. This could give an explanation why the speed of the furrow is reduced when it reaches the proximity of the apical membrane.

Another question concerns how the gap closes basally. The concentration of cytokinetic molecules at the tip of the inverted teardrop could be involved in membrane spreading. Reversely, the lower density of cytokinetic molecules on the basal side of the inverted teardrop could lead to membrane relaxation allowing subsequent daughter cells contacts. However, we cannot exclude the possibility that an active process could also be involved in the gap closure. Obviously, the teardrop is not empty. It will be interesting to determine how it is filled and if this participates in producing and or maintaining the teardrop shape.

Asymmetric furrowing is potentially important to maintain the epithelial integrity, homeostasis and function during cell division. Our present study shows that the two daughter cells enter in contact basally to the gap while division is still going on, thus closing the gap. This mechanism probably contributes to maintain the epithelial integrity by ensuring cell-cell cohesion while cytokinesis is not completed. Consequently, the gap is transient and the two daughter cells are in close contact. However, we show that this general scheme has an exception which is intercalation of neighboring cells. Although it may not be the unique mechanism, we show here that intercalation of neighboring cells during cytokinesis participate in producing cell mixing during Xenopusgastrulation. In contrast with other model organisms such as zebrafish and mouse in which massive cells mixing happens during embryogenesis, in Xenopus cell mixing is restricted to clone borders (Bauer and Moody, 1994). This limited cell mixing explains why the Xenopus fate map is consistent. It was hypothesized that intercalation could take place by interdigitation of neighboring cells during mitosis. Our results show that this phenomenon indeed occurs and produces cell mixing. Interestingly, a previous electron microscopy study performed with mouse small intestine showed that neighboring cells could infiltrate the space in the cytokinetic furrow (Jinguji and Ishikawa, 1992). This indicates that some features of the relationships between the dividing cells with their neighbors such as those reported here could be present in mammalian tissues. Our results using the Xenopus embryo as a model system to study cell division also provide a more precise view on interactions between daughter epithelial cells during cytokinesis in vivo.

\section{Materials and Methods}

\section{Preparation of Xenopus embryos}

Xenopus laevis albinos were obtained from Biological Resources Centre (CRB, Rennes, France). Xenopus laevis embryo were collected and fertilized as described previously (Chartrain et al., 2013).

\section{Microinjection of in vitro transcribed mRNAs}

pT7T-SF9-GFP was obtained by PCR amplification of SF9-GFP intrabody (Nizak et al., 2003) and subcloned into the pT7T vector. pCS2GFP-GPI, pCS2-mRFP-GPI plasmids (both kindly provided by Michael
Brand), pT7T-GFP-anillin (Le Page et al., 2011), pCS2-mcherry-Utr-CH (Burkel et al., 2007) and pT7T-SF9-GFP were linearized and mRNAs were synthesized in vitro with the SP 6 or T7 mMessage mMessage transcription kit according to the manufacturer's instructions (Ambion). For microinjection, embryos were placed in $5 \%$ of Ficoll in $\mathrm{F} 1$ medium (10 mM HEPES $\mathrm{pH}$ 7.6, $31.2 \mathrm{mM} \mathrm{NaCl}, 1.75 \mathrm{mM} \mathrm{KCl}, 59 \mu \mathrm{M} \mathrm{MgCl}_{2}, 2 \mathrm{mM} \mathrm{NaHCO}_{3}, 0.25$ $\mathrm{mM} \mathrm{CaCl} 2$ ). mRNAs were microinjected into one blastomere of 2 cells stage embryos. To visualize the border between cells, mRNAs encoding GFP-GPI and RFP-GPI were injected respectively into blastomeres in 2 of 4 cells stage embryos.

\section{Indirect immunofluorescence}

Indirect immunofluorescence was performed essentially as described previously (Le Page et al., 2011). Briefly, gastrula (stage 11, Nieuwkoop and Faber, 1994) were fixed with $2 \%$ trichloroacetic acid (TCA) in F1 medium for 2 hours at room temperature. Embryos were devitellinated, permeabilized in PBS (137 mM NaCl, $2.7 \mathrm{mM} \mathrm{KCl}, 8 \mathrm{mM}$ Na2HPO4, $2 \mathrm{mM}$ $\mathrm{KH} 2 \mathrm{PO} 4, \mathrm{pH} 7.4$ ) plus $1 \%$ Triton $\mathrm{X}-100$ for 20 minutes at room temperature, incubated in PBS plus $0.1 \%$ Trtiton X-100 (PBST $0.1 \%$ ) for 10 minutes and then 1 hour in $2 \%$ BSA in PBST $0.1 \%$. Embryos were incubated with affinity-purified rabbit anti-xMELK (Blot et al., 2002, Le Page et al., 2011) final concentration $1 \mu \mathrm{g} / \mathrm{ml}$ in $2 \%$ BSA in PBST $0.1 \%$ overnight at $14^{\circ} \mathrm{C}$. After washing with PBST $0.1 \%$, embryos were incubated with Alexa Fluor 555 coupled anti-rabbit immunoglobulin secondary antibody (Molecular Probes, 1:200) and TO-PRO-3 to stain DNA (Invitrogen, $0.5 \mu \mathrm{g} / \mathrm{ml}$ ) in $2 \%$ BSA in PBST $0.1 \%$ for 1 hour at room temperature. Embryos were washed in PBST $0.1 \%$ and whole-mounted between a glass slide separated from a glass coverslip by 2 stacked hybridization frames (65 $\mu$, Gene Frame) in Vectashield (Vector).

\section{Imaging}

Living embryos were mounted in an observation chamber constituted of a glass slide separated from a glass coverslip by 3 stacked hybridization frames (65 $\mu \mathrm{l}$, Gene Frame) filled with $5 \%$ of Ficoll in F1 medium. This montage limits the pressure applied onto embryos during microscopic observations. Imaging was performed using a Leica SP5 confocal microscope with a 40x HC Plan-APO- ON 1.25 oil immersion objective lens or a Leica SPE, 63x HCX PL APO- ON 0.75 oil immersion objective lens (Microscopy Rennes Imaging Center, MRic). Images were processed using ImageJ software (Rasband, W.S., http://rsb.info.nih.gov/ij) and figures were prepared using ImageJ and Photoshop.

\section{Acknowledgments}

We thank Michael Brand, William M. Bement, Franck Perez for plasmid constructs, the Cell Cycle Team and Roland Le Borgne for helpful discussion. We also thank the Microscopy Rennes Imaging Center (MRic, BIOSIT, IBISA). This work was supported by l'Agence Nationale de la Recherche (ANR, KinBioFRET) and le Centre National de la Recherche Scientifique (CNRS).

\section{References}

ALMONACID M, PAOLETTIA(2010). Mechanisms controlling division-plane positioning. Semin Cell Dev Biol 21: 874-880.

AUDHYAA, HYNDMAN F, MCLEOD I, MADDOX AS, YATES JR 3RD, DESAI A OEGEMA K (2005). A complex containing the Sm protein CAR-1 and the RNA helicase $\mathrm{CGH}-1$ is required for embryonic cytokinesis in Caenorhabditis elegans. J Cell Biol 171: 267-279.

BAUER DV, HUANG S, MOODY SA (1994). The cleavage stage origin of Spemann's Organizer: analysis of the movements of blastomere clones before and during gastrulation in Xenopus. Development 120: 1179-1189.

BLOT J, CHARTRAIN I, ROGHI C, PHILIPPE M, TASSAN JP (2002). Cell cycle regulation of $\mathrm{pEg} 3$, a new Xenopus protein kinase of the KIN1/PAR-1/MARK family. Dev Biol 241: 327-338.

BURKEL BM, VON DASSOW G, BEMENT WM (2007). Versatile fluorescent probes 
for actin filaments based on the actin-binding domain of utrophin. Cell Motil Cytoskeleton 64: 822-832.

BYERSTJ,ARMSTRONG PB (1986). Membrane protein redistribution during Xenopus first cleavage. J Cell Biol 102: 2176-2184.

CHARTRAIN I, LE PAGE Y, HATTE G, KORNER R, KUBIAK JZ, TASSAN JP (2013). Cell-cycle dependent localization of MELK and its new partner RACK1 in epithelial versus mesenchyme-like cells in Xenopus embryo. Biol Open 2: 1037-1048.

CRAMER LP, MITCHISON TJ (1997). Investigation of the mechanism of retraction of the cell margin and rearward flow of nodules during mitotic cell rounding. $\mathrm{Mol}$ Biol Cell 8: 109-119.

FEDEDA JP, GERLICH DW (2012). Molecular control of animal cell cytokinesis. Nat Cell Biol 14: 440-447.

GREEN RA, PALUCH E, OEGEMA K (2012). Cytokinesis in animal cells. Annu Rev Cell Dev Biol 28: 29-58.

HERSZTERG S, LEIBFRIED A, BOSVELD F, MARTIN C, BELLAICHE Y (2013). Interplay between the dividing cell and its neighbors regulates adherens junction formation during cytokinesis in epithelial tissue. Dev Cell 24: 256-270.

JINGUJI Y, ISHIKAWA H (1992). Electron microscopic observations on the maintenance of the tight junction during cell division in the epithelium of the mouse small intestine. Cell Struct Funct 17: 27-37.

KELLER R (2002). Shaping the vertebrate body plan by polarized embryonic cell movements. Science 298: 1950-1954.

KITTLER R, PUTZ G, PELLETIER L, POSER I, HENINGER AK, DRECHSEL D, FISCHER S, KONSTANTINOVA I, HABERMANN B, GRABNER H, YASPO ML, HIMMELBAUER H, KORN B, NEUGEBAUER K, PISABARRO MT, BUCHHOLZ $F$ (2004). An endoribonuclease-prepared siRNA screen in human cells identifies genes essential for cell division. Nature 432: 1036-1040.
KUBIAK JZ, CHARTRAIN I and TASSAN JP (2013). The Xenopus embryo as a mode system to study asymmetric furrowing in vertebrate epithelial cells. In Xenopus Development (Eds Kloc and Kubiak). John Wiley \& Sons, Inc, Oxford. pp 103-111.

LE PAGE Y, CHARTRAIN I, BADOUEL C, TASSAN JP (2011). A functional analysis of MELK in cell division reveals a transition in the mode of cytokinesis during Xenopus development. J Cell Sci 124: 958-968.

LECUIT T, WIESCHAUS E (2000). Polarized insertion of new membrane from a cytoplasmic reservoir during cleavage of the Drosophila embryo. J Cell Biol 150: 849-860.

MADDOX AS, BURRIDGE K (2003). RhoA is required for cortical retraction and rigidity during mitotic cell rounding. J Cell Biol 160: 255-265.

MERZDORF CS, CHEN YH, GOODENOUGH DA (1998). Formation of functional tight junctions in Xenopus embryos. Dev Biol 195: 187-203.

NIEUWKOOP PD and FABER J (1956). In Normal table of Xenopus laevis (Daudin) Garland Publishing Inc, New York

NORMAND G and KING RK (2010). Understanding cytokinesis failure. Adv Exp Med Biol 676: 27-55.

NIZAK C, MARTIN-LLUESMAS, MOUTELS, ROUXA, KREIS TE, GOUD B, PEREZ $F$ (2003). Recombinant antibodies against subcellular fractions used to track endogenous Golgi protein dynamics in vivo. Traffic 4: 739-753.

PIEKNY AJ, MADDOX AS (2010). The myriad roles of Anillin during cytokinesis. Semin Cell Dev Biol 21: 881-891.

RAPPAPORT R (1996). In Cytokinesis in Animal Cells (Developmental and Cell Biology Series 32) Cambridge Univ. Press, Cambridge, UK.

WOOLNER S, PAPALOPULU N (2012). Spindle position in symmetric cell divisions during epiboly is controlled by opposing and dynamic apicobasal forces. Dev Cell 22: 775-787. 


\section{Further Related Reading, published previously in the Int. J. Dev. Biol.}

Sexual dimorphism of AMH, DMRT1 and RSPO1 localization in the developing gonads of six anuran species

Rafal P. Piprek, Anna Pecio, Katarzyna Laskowska-Kaszub,Jacek Z. Kubiak and Jacek M. Szymura

Int. J. Dev. Biol. (2013) 57: 891-895

Dual embryonic origin of the hyobranchial apparatus in the Mexican axolotl (Ambystoma mexicanum)

Asya Davidian and Yegor Malashichev

Int. J. Dev. Biol. (2013) 57: 821-828

Clonal analyses in the anterior pre-placodal region: implications for the early lineage bias of placodal progenitors

Sujata Bhattacharyya and Marianne E. Bronner

Int. J. Dev. Biol. (2013) 57: 753-757

Amphibian interorder nuclear transfer embryos reveal conserved embryonic gene transcription, but deficient DNA replication or chromosome segregation

Patrick Narbonne and John B. Gurdon

Int. J. Dev. Biol. (2012) 56: 975-986

Origins of Cdx1 regulatory elements suggest roles in vertebrate evolution

Stephen J. Gaunt and Yu-Lee Paul

Int. J. Dev. Biol. (2011) 55: 93-98

Reptile scale paradigm: Evo-Devo, pattern formation and regeneration

Cheng Chang, Ping Wu, Ruth E. Baker, Philip K. Maini, Lorenzo Alibardi and Cheng-Ming Chuong

Int. J. Dev. Biol. (2009) 53: 813-826

Proteomics analysis of regenerating amphibian limbs: changes during the onset of regeneration

Michael W. King, Anton W. Neff and Anthony L. Mescher

Int. J. Dev. Biol. (2009) 53: 955-969
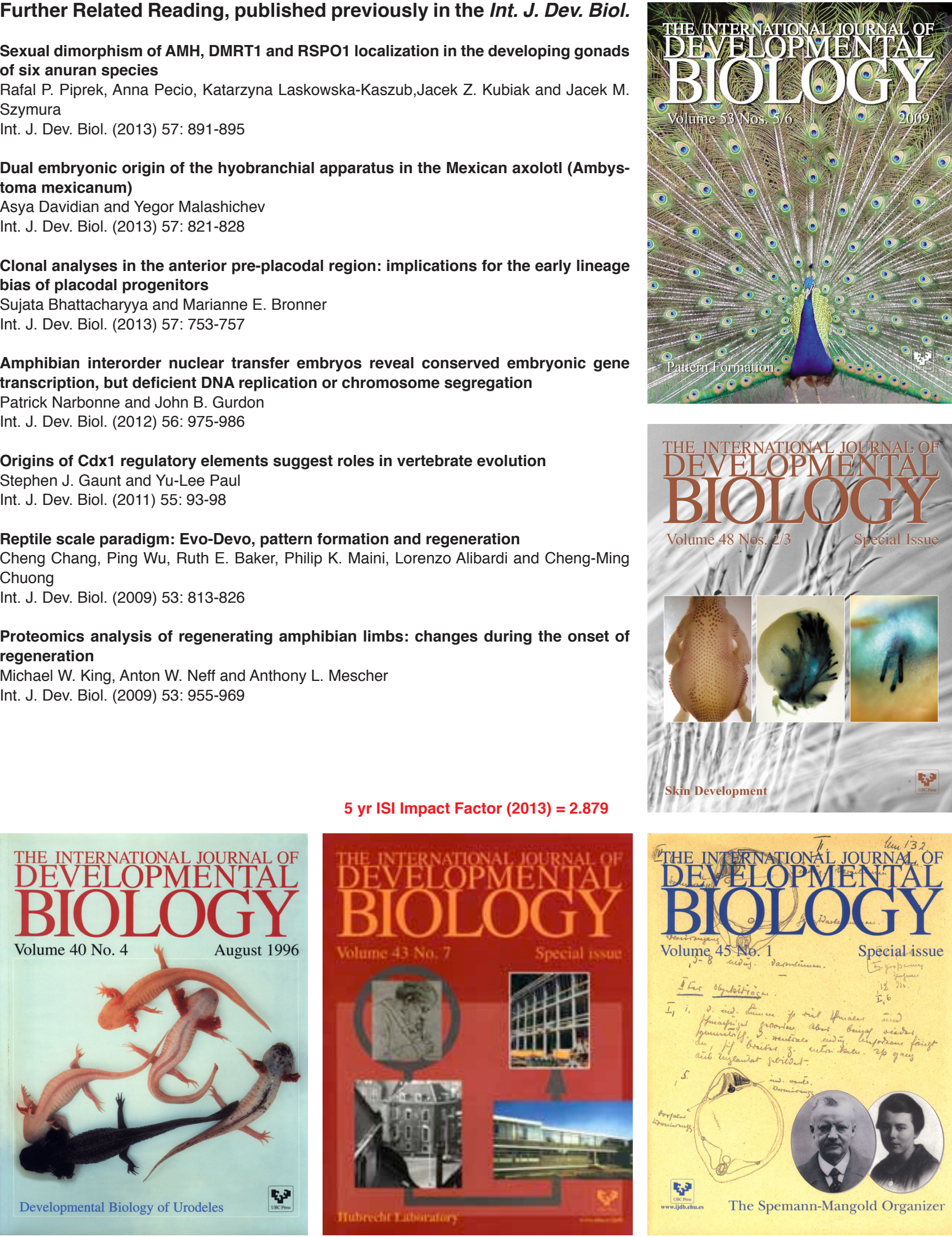